\title{
SCRIVERE IL FUTURO: 1957-1967. TECNICA E IDEOLOGIA NELLA CRITICA D'ARTE TRA ROMA E BELGRADO
}

\begin{abstract}
Dal 1957 al 1967 diversi episodi nel sistema dell'arte contemporanea italiana e ex jugoslava furono in relazione con la produzione industriale e la cosiddetta ideologia della tecnica. Le teorie sul progresso marxiste incontrarono la riflessione sul rapporto uomo-macchina. Si diffuse un pensiero comune, e in alcuni casi condiviso, tra artisti, storici e studiosi che tentarono una comprensione dei nuovi fermenti artistici. In particolare critici d'arte italiani, come Giulio Carlo Argan, Umbro Apollonio e Giuseppe Gatt, e studiosi di Belgrado, come Oto Bihalji-Merin, Lazar Trifunović e Jerko Denegri, si interessarono al rapporto tra arte cinetica/programmata e tecnologia: ciò da un lato si manifestò nella serie di esposizioni internazionali tenutesi a Zagabria, che ebbero il titolo di Nove tendencije e dall'altro prese corpo grazie alle nuove forme del disegno industriale. I critici ebbero l'ambizione di tracciare la rotta ideologica delle ricerche delle nuove tendenze, ma fallirono alla prova dei fatti storici. Un'analisi delle parole e dei concetti impiegati nella critica d'arte italiana e serba, entrambe coadiuvate da quella croata, non è stata ancora svolta con metodo scientifico e questo saggio non mira ad esaurire l'argomento ma ad avviare un dibattito storico-critico di respiro internazionale.

Parole chiave: tecnica, macchina, arte cinetica, disegno industriale, Nove tendencije, ideologia, critica d'arte
\end{abstract}

\section{NOTIZIE DAL MONDO FUTURO: ECONOMIA, POLITICA E CULTURA}

Dalla fine degli anni Cinquanta le relazioni tra Italia e Jugoslavia si rafforzarono nella cooperazione economica, grazie ai commerci e agli scambi di materie prime (Vrsaj 1970). Sul versante politico, l'intellighenzia italiana, lontana dal Partito Comunista, sostenne la fase del revisionismo jugoslavo (Olivetti 1959).

*giovanni.rubino_78@libero.it 
A livello culturale, un comune tema fu il rapporto tra creatività umana e macchine. Giulio Carlo Argan (1953) difendeva il disegno industriale come possibilità di dialogo tra arte e industria mentre Oto Bihalji-Merin (1970) sosteneva che il mondo tecnologico influenzasse a livello simbolico la creazione artistica. Su tali problematiche i primi luoghi di confronto, che coinvolsero altri studiosi italiani e serbi, furono le esposizioni internazionali Nove tendencije ${ }^{1}$ a Zagabria nel 1963 e 1965, curate da Radoslav Putar e Matko Meštrović.

Nell'edizione del 1963, le teorie sull'arte come indagine scientifica si fusero con l'ideologia marxista di critici ed artisti, che ambivano a rivoluzionare la vita nel quotidiano. La successiva edizione nel 1965, tuttavia, secondo Donald Drew Egbert (1970), fu contro-rivoluzionaria poiché si era assistito alla istituzionalizzazione delle nuove tendenze e ad una neutralità politica conveniente per il Partito Comunista jugoslavo. Similmente, lo studioso Piotr Piotrowski (2009) ha sostenuto che il governo jugoslavo avesse tollerato le nuove tendenze per puro opportunismo politico. D'altra parte Jerko Denegri (2003) ha rivalutato l'azione svolta dalle nuove tendenze perché avrebbero consentito di costruire un via propriamente jugoslava al "modernismo socialista".

Effettivamente dopo il 1967 il ruolo rivoluzionario ricadde su altre ricerche artistiche (Medosh 2010) che, come l'Arte povera, avrebbero attuato nuove strategie contro la supremazia della tecnica. Nel corso degli anni Sessanta, tuttavia, si erano imposte parole come sperimentale, macchina, nuovo, disegno industriale, produzione, anonimato e collettivo, cui si aggiunsero: struttura, ottico, cinetico, programmato, Gestalt, tecnologia e cibernetico, che divennero il comune armamentario della critica d'arte in Italia e in Croazia. Le stesse parole, tuttavia, anche in Serbia furono impiegate dalla critica che, complice l'apertura di Belgrado alla scena internazionale dell'arte (Denegri 2009), ebbe inediti scambi con le nuove tendenze italiane e croate.

${ }^{1}$ La pubblicazione internazionale più recente è il volume curato da Margit Rosen (2010) e pubblicato dallo Zenter für Kunst und Medientechnologie di Karlsruhe, in concomitanza con l'esposizione BIT international [nove] tendencije Computer und visuelle Forschung. Zagreb 1961-1973 del 2009. Si ricordano i saggi di Jerko Denegri e Želimir Koščević (1979) e del solo Denegri (2001). Uno studio sulle relazioni tra Italia e Jugoslavia, nonché un'ampia bibliografia, è il tema del mio progetto di ricerca per il dottorato in Storia dell'arte svolto presso l'Università di Udine, in cotutela con l'Università di Zagabria. Testo disponbile al sito https://www.dspace-uniud.cineca.it. 


\section{LA TECNICA: FARE DELLA MACCHINA UN'OPERA D'ARTE}

Nel 1958 l'esposizione universale di Bruxelles permise l'incontro tra l'Ovest e l'Est del mondo, su di un comune terreno di scambi scientifici, artistici e tecnologici. Emerse la celebrazione della tecnica, dell'industria e delle macchine, considerati veicoli per la pace fra i popoli nel clima drammatico della Guerra Fredda (Devos \& De Kooning 2006).

Nella cosiddetta "crisi" degli anni Cinquanta (Jeannesson, 2002/2003), Lewis Mumford (1952), in modo ottimistico, sosteneva che la creatività e la tecnica avrebbero portato la bellezza nell'oggetto industriale e nella vita quotidiana. Herbert Read (1961), inoltre, considerava la relazione tra artista e industria come motore per il progresso sociale. All'opposto, Sigfried Giedion (1948) avanzava il sospetto che le macchine stessero manipolando l'umanità.

Nella pittura si sviluppò una polarità tra l'espressione dell'irrazionale e l'estetica dell'ingegneria come rappresentazioni dell'esistenza umana (Neumeyer 1964). Da una parte la pittura Informale era la manifestazione di una romantica ribellione e isolamento mentre, dall'altra, l'estetica ingegneristica riportava l'artista al suo impegno sociale. Nella nuova dimensione scientifica e tecnica della società, dopo il 1960, infatti, sulla scena internazionale le nuove ricerche di artisti singoli e riunitisi in gruppi ponevano un problema fondamentale: come un oggetto potesse avere valore estetico anche se composto da parti meccaniche ed elettriche e non dai materiali della tradizione.

\subsection{Astrazione Informale vs. Astrazione Geometrica}

"Il 'culto della personalità', in arte come in politica, sostituendo il principio d'autorità a quello della libera ricerca e della libera scelta, ha sempre rappresentato il freno principale a ogni movimento dell'intelletto umano [...]. La scoperta dell'energia atomica, e la messa in atto dei postulati del Socialismo, aprono una nuova era all'umanità" (Sauvage 1957: 13, 24). Nel 1957 con queste parole - non estranee alla condanna di Stalin avvenuta nel 1956 - il critico d'arte italiano Tristan Sauvage (alias Arturo Schwarz) sintetizzava il rapporto tra arte e società nella pittura del secondo dopoguerra.

Il suo discorso s'inseriva nel panorama di una doppia articolazione delle ricerche artistiche: la tradizione astratta e geometrica, che trasponeva il modus operandi della macchina, quindi il massimo di anonimato, si opponeva alla corrente pittorica cosiddetta 'informale' o 'tachiste', che privilegiava l' 'io' del pittore, quindi il massimo di individualità. Entrambe in Europa erano al centro degli interessi degli artisti, dei critici e dei galleristi 
(Dorfles 1961), poiché l'astrazione, razionale o irrazionale che fosse, era il vessillo della modernità.

La Jugoslavia, dopo l'allontanamento dai dogmi del realismo socialista (Wachtell 1998), era stata accolta nella cultura occidentale ma l'esistenzialismo della pittura informale si accompagnava ad una crisi morale. A Belgrado, dove nel 1955 era nato il Decembarska grupa con l'intento di attualizzare le ricerche verso l'astrazione delle forme (Merenik 2010), lo storico dell'arte Lazar Trifunović sul giornale Delo nel 1957 scrisse: "I jugoslovensko posleratno slikarstvo gotovo da u malome sadrži osnovnu tendenciju evropskog pedesetgodišnjeg procesa. Ono je započelo jednom ozbiljnom krizom (socijalističkim realizom) i reakcijom na tu krizu, koja se usmerila nefigurativnoj koncepciji, oštroj deformaciji oblika i težnji ka negaciji realne forme" (Trifunović 1990: 162)².

Le idee di Trifunović rispecchiavano una situazione molto particolare in cui la pittura belgradese si stava aggiornando nella scuola dell'informale, a sostegno delle cui ragioni - autonomia e libertà creativa - si preferiva privilegiare l'individualità dei singoli artisti, interpretandola come "moderno naturalismo" in opposizione agli anni grigi del realismo socialista (Trifunović 1990: 151).

Nella cultura democratica e liberale italiana, invece, Argan attestava che dopo un decennio di 'accademia' dell'informale, la ricerca era scaduta in una sfera anti-ideologica rispetto all'avanguardia storica. L'individualismo dell'informale si differenziava dal tecnicismo industriale perché non aveva "una direzione costante di ricerca, una regola operativa, un fine utilitario" (Argan 1959: 5) che, all'opposto, si ritrovavano nella spersonalizzazione dell'astrazione geometrica.

\subsection{La funzione tecnica dell'arte: estetica del disegno industriale}

Nel 1958 Oto Bihalji-Merin collaborò con Argan nella pubblicazione L'arte dopo il 1945. La pittura, tradotta in Italia nel 1959, per illustrare l'evoluzione dell'arte tra l'Europa Occidentale e Orientale (Jugoslavia e Polonia). Nella pittura italiana Argan aveva evidenziato la presenza del gruppo Forma (nato nel 1948) che produceva opere astratte e allo stesso tempo, seguendo un comune pensiero marxista, era impegnato politicamente nella

2 "La pittura jugoslava del dopoguerra contiene in nuce la tendenza base dello sviluppo europeo dell'ultimo cinquantennio. È iniziata una grave crisi (realismo socialista), e la reazione a questa crisi ha mirato alla concezione non-figurativa, all'acuta deformazione della forma con l'aspirazione alla negazione delle forme reali". (Le traduzioni in italiano nelle note a piè di pagina sono dell'autore.) 
società: "[...] occorreva infatti riportare alla discussione il concetto stesso del linguaggio artistico [...] dichiarare che i propri contenuti, sorti da una nuova condizione, dovevano essere realizzati nell'ambito di una visione che non si preoccupasse della riproduzione naturalistica. Tutto questo era dovuto $[\ldots]$ all'idea di ritrovare una razionalità estranea ad ogni romanticismo [...] alla fiducia che $[. .$.$] si poteva esprimere quella che era la nuova condizione$ storica e sociale dell'uomo" (Argan \& Ponente 1959: 89-90).

Complementare era la posizione di Bihalji-Merin nell'ambito dell'estetica ingegneristica e del ruolo sociale dell'artista. La nuova situazione jugoslava era spiegata nel rapporto tra arte e tecnica, chiamando in causa la situazione croata degli anni Cinquanta:

\begin{abstract}
"Dopo che nel mondo orientale della riforma socialista l'arte ufficiale era stata avviata verso le mete di un primitivo conformismo materialistico e di un naturalismo deteriore, in Iugoslavia; [...] la coscienza e le aspirazioni artistiche si sono volte di nuovo, liberamente, ai problemi e agli svolgimenti dell'arte moderna. [...] Con un senso dell'oggettivo privo di ogni traccia di passionalità, giovani pittori e architetti come Ivan Picelj, Bozidar Rašica, Aleksandar Srnec e altri, riducono il dipinto a quella concretezza di forma, priva di un contenuto ben definito, che nasce dal nesso architettonico dello spazio" (Bihalji-Merin 1958/1959: 132, 134).
\end{abstract}

Il diretto riferimento era al gruppo EXAT51 di Zagabria, che era stato in mostra anche a Belgrado nel 1953. EXAT51 aveva ripreso l'astrazione geometrica, affermando un'assoluta parità estetica e progettuale tra l'arte del sistema tradizionale e gli oggetti prodotti dall'industria (Denegri \& Koščević 1979).

Si presentava la questione fondamentale riguardante la possibilità che la macchina producesse opere considerabili arte. La progettazione dell'oggetto trasformava l'artista in designer, che non doveva essere subordinato all'industria, ma per mezzo di essa doveva concorrere al benessere della società.

Simili concetti vennero poi ripresi anche da Argan, in linea col pensiero marxista, nel giugno 1961: "La possibilità di educare o formare o riformare la società through design, [...] era subordinata al fatto che l'artista-progettista potesse controllare e orientare lo sviluppo progressivo della tecnica e, in un ambito più largo, il comportamento attivo o produttivo della società: ciò che significa assumere la direzione politica della produzione" (Argan 1961: 5).

Herbert Read, Sigfried Giedion, Lewis Mumford, oltre Karl Marx, erano un importante trait d'union tra il pensiero di Argan e di Bihalji-Merin. Bihalji-Merin teorizzava una nuova estetica, incentrata sul rapporto costruttivo tra creatività e civiltà tecnologica:

"Kasnograđanski individualizam potpuno je odvojio razne grane umetnosti, raskinuo društvene veze umetnika i zajednice, ili ih redukovao na subjektivno opštenje 
umetnika sa uskim krugom svoje grupe. Razdvanje arhitekture, skulpture i slikarstva isto je što i rascep svesti; ono razbija jedinstvenost estetike - uslov totalnog umetničkog dela. [...] U dvadesetom veku konstrukcija se osamostalila i, obnažavanjem funkcije, pretvorila u umetnički izraz. Za takvu proširenu estetsku svet može naučna aparatura ili tehnička konstrukcija i sama da deluje stvaralački i savršeno kao pravo umetničko delo našeg vremena" (Bihalji-Merin 1962: 129, 130)³.

Nel medesimo saggio, il legame tra "struttura" artistica e "struttura" tecnica si richiamava inoltre al filosofo e docente di estetica a Ulm, Max Bense (1960) - conosciuto anche da Argan e da Meštrović - che nel quarto libro della sua Aestetika aveva rielaborato il concetto scientifico del materialismo storico marxista. Applicando la teoria dell'informazione ai fenomeni ghestaltici, secondo Bense, si poteva programmare un'oggettiva percezione estetica in sintonia con la nascente scienza della cibernetica.

Se tali erano i presupposti in Italia e Jugoslavia, le diverse generazioni di artisti, grazie ad una rete di relazioni a livello europeo, pur ricercando autonomamente, finirono per convergere nella prima edizione di Nove tendencije del 1961 (Denegri 2001). S'incontrarono da una parte alcuni fondatori del gruppo EXAT51 e dall'altra i più giovani artisti del gruppo $\mathrm{N}$ (Biasi, Chiggio, Landi, Massironi), proveniente da Padova e di orientamento marxista (Feierabend \& Meloni 2009). Entrambe le formazioni rivendicavano un ruolo politico e sociale per l'artista e l'applicazione all'industria delle proprie ricerche. L'approccio scientifico alla creazione artistica, di Picelj, Srnec e del Gruppo N, ebbe come modello la teoria della Gestalt. La riduzione delle forme alla loro struttura e le relazioni fra le parti oggettivamente calcolabili si ponevano in relazione con l'osservatore, ridotto ad essere un mero recettore ottico.

\section{L'IDEOLOGIA: GLI ARTISTI AL SERVIZIO DELLA SOCIETÀ}

I processi e le finalità delle opere d'arte potevano essere 'programmati', come divenne evidente nel maggio 1962 con la mostra Arte programmata (Meneguzzo 2012), finanziata e promossa dall'azienda Olivetti. Le opere

3 'L'individualismo tardo borghese ha completamente separato i vari rami dell'arte, recidendo i legami sociali di artisti e comunità, o ridotto loro alla soggettiva comunicazione dell'artista con la ristretta cerchia del proprio gruppo. La divisione di architettura, scultura e pittura è come una coscienza divisa. Si rompe l'unità della condizione estetica dell'opera d'arte totale.[...] Nel XX secolo la struttura è indipendente e, rivelando le funzioni, trasformata in espressione artistica. Per tale diffusa estetica, il mondo dell'apparato scientifico o della struttura tecnica può da solo agire in modo creativo e perfetto come diretta opera d'arte del nostro tempo". 
esposte erano formalmente assimilabili ai prodotti industriali: le calcolatrici da tavolo, le macchine da scrivere nonché i primi cervelli elettronici. L'arte programmata poteva considerarsi come la manifestazione di un'estetica tecnologica (Bricco 2005).

Arte programmata e simili esposizioni che seguirono in Italia e in Europa furono al centro di un acceso dibattito estetico e politico che si riverberò anche in Jugoslavia nella seconda edizione di Nove tendencije 2 del 1963. Argan era a capo dei sostenitori della cosiddetta "arte ghestaltica" (calco dalla parola tedesca Gestalt, impiegata nella psicologia della percezione).

La relazione tra arte e industria avveniva entro il sistema della progettazione industriale che, come suggerito da Meštrović, avrebbe garantito all'artista di diventare un tecnico, la cui professionalità si esercitava nel campo degli oggetti quotidiani. Quando da Zagabria la mostra arrivò a Venezia, intitolata Nuova tendenza 2, il critico d'arte Umbro Apollonio (1963a) sostenne che l'arte non sarebbe stata subordinata alla tecnica e alla scienza, ma grazie ad esse avrebbe opposto un principio di rigore all'assoluta libertà dell'informale. Il rischio, tuttavia, era che questi nuovi oggetti artistici sembrassero riproporre un'estetica che ricalcava le forme del costruttivismo storico, perdendo così il contatto con l'azione diretta nel mondo.

\subsection{Scienza e marxismo nell'opera d'arte ghestaltica}

Nel settembre del 1963 Argan aveva individuato la finalità dell'arte ghestaltica nell'impegno sociale dell'artista: "Le ricerche 'ghestaltiche' condotte da gruppi di artisti di vari paesi [...] muovono [...] dallo studio dei processi psicologici e, più precisamente, dai risultati della 'psicologologia della Gestalt'. [...] Lo scopo è di contenere l'inflazione delle immagini che minaccia di soffocare e paralizzare le facoltà imago poietiche dell'individuo o di indirizzarlo a una falsa finalità" (Argan 1963a: 3).

Argan, in altro luogo, aveva già espresso il suo rammarico per il cattivo rapporto che le arti intrattenevano con la scienza, e prevedeva non una fusione tra arte e scienza ma che l'arte compensasse, a livello qualitativo, il progresso scientifico: "[...] la corrente costruttivista mira a risolvere tutte le operazioni artistiche nella progettazione (si tratti di architettura, di pittura, di scultura o di 'disegno industriale') [...] di qui la sua importanza nella formazione e nello sviluppo del Disegno Industriale [...] Ma la doppia polarità dialettica dell'arte contemporanea non è [...] l'antitesi di astrattismo e realismo: è invece l'antitesi [...] di tecnologia e ideologia" (Argan 1963b: 109). 
Pochi mesi prima, infatti, l'analisi storiografica di Apollonio sulla rivista Quadrum, aveva evidenziato la continuità tra l'avanguardia storica costruttivista e le ricerche successive, e auspicava l'insorgere di un nuovo stile, comune agli artisti delle nuove tendenze, di valenza internazionale.

"Quindi che la tecnica e la scienza possono fornire contenuti all'arte alla pari di altre discipline e che esse possono anche contenere valori gnoseologici non soltanto passibili ma degni di essere comunicati. [...] le varie sfere creative finiscono con il comunicare fra loro e si compongono in un'entità dove non sono più distinguibili perché omogeneizzate, è giusto che si sperimenti la possibile cooperazione di scienza ed arte, di tecnica e fantasia, in vista di una partecipazione totale al determinare una situazione. [...] Dove la parola 'stile' possa [...] significare unitarietà di interessi[...]" (Apollonio 1963b: 23, 34).

Questa situazione di osmosi tra le arti visuali e il design non sfuggì all'occhio critico di Trifunović che su Politika nel 1964 scrisse:

"[...] industrijsko oblikovanje ima dva osnovna plana: unutrašnji i spoljašnji. Unutrašnji se odnosi na stvaralaštvo i metode realizacije [...], dok je spoljašnji plan tesno povezan sa sociološkom ulogom dizajna: da u čoveku probudi [...] smisao za nove harmonije, da izmeni njegovu psihologiju i prilagodi je novom industrijskom vremenu. [...] Dizajn nije budućnost umetnosti (i na tom planu otpadaju sve polemike) već jedan dugotrajni proces koji treba da izmeni sliku sveta [...]. Time će ujedno stvoriti u čoveku plodno tle i za ono što zovemo klasična umetnost" (Trifunović 1990: 175) ${ }^{4}$.

Trifunović, pur procedendo attraverso un'attenta analisi atta ad evidenziare l'aderenza tra le forme industriali e il nuovo mondo tecnologico, volle marcare un principio di eterogeneità delle arti piuttosto che sostenere una pericolosa omogeneità ideologizzata.

\subsection{Tecnica e ideologia: il materialismo storico e il ruolo dell'artista}

Rispetto a Trifunović, le posizioni di Argan e Apollonio trovarono un singolare parallellismo nell'articolo scritto da un altro storico e critico d'arte serbo Dragoslav Đorđević, che sul giornale Borba, richiamando l'attenzione

4 "Il disegno industriale ha due liveli basilari: interno ed esterno. L'interno è legato alla creatività e metodi di attuazione [...], mentre l'esterno è strettamente legato con il ruolo sociologico del design: risvegliare nell'uomo un senso di armonia nuovo, modificare la sua psicologia e adattarsi al nuovo tempo industriale. [...] Il design non è il futuro dell'arte (e ciò elimina ogni controversia) ma un lungo processo che deve cambiare l'immagine del mondo [...]. Questo creerà anche un terreno fertile nell'uomo e per ciò che chiamiamo arte classica". 
su Zagabria, storicizzava le nuove tendenze e ne evidenziava il rapporto con la tecnologia e l'architettura:

"Još 1951. u Zagrebu je formirana umjetnička grupa, tzv. Eksperimentalni atelje 'Exat 51' [...] Među članovima grupe nailazimo imena arhitekta Vjenceslava Richtera [...] i slikara Ivana Picelja, Aleksandra Srneca i Vlade Kristla, od kojih su poslednja četvorica 1953. priredili izložbu slikarskih radova u [...] Beogradu. [...] Ta tradicija [...] stvorila je pogodnu platformu za njeno uključivanje u šire, međunarodne okvire [...]. Ovako scientificirani umjetnički predmeti gube svoj 'unikatni' karakter i mogu se lako pretvoriti u produkte mašinske, tvorničke proizvodnje, što svakako utječe na mogućnost njihovog širokog plasiranja i niže prodajne cijene. [...] Ako se uvjetno prihvate ove nove forme umjetničkog izražavanja, ili nazovino ih kako hoćemo, onda njihovu budućnost treba tražiti u njihovoj sintezi s arhitekturom [...]" (Đorđević 1963: 6) .

In Italia un evento che coinvolse le nuove tendenze fu il XIII Convegno di artisti, critici e studiosi d'arte, tenutosi a Rimini nel settembre 1964. Dedicato al tema Tecnica e ideologia, vi presero parte, tra gli italiani, Argan e Giuseppe Gatt, e Aleksa Čelebonović e Meštrović tra gli invitati jugoslavi. L'interesse dei colleghi jugoslavi alla questione della tecnica, nelle parole di Argan (1964: 33), non implicava che questi fossero integrati al sistema, ma che non vi era l'urgenza di un impegno ideologico, poiché era stato in gran parte "soddisfatto" grazie alla loro attuale situazione politica.

Il critico d'arte Gatt scrisse un articolo incentrato sugli scambi tra arte, tecnologia e ideologia. Gatt suffragava, in accordo con le teorie di Argan, che l'arte programmata era positiva nei confronti delle relazioni tra produzione industriale e cultura scientifica: "[...] esiste attualmente una forma d'arte a carattere attivo e positivo e che mira esattamente a penetrare nei processi produttivi dell'industria per tentarne, quanto meno, un condizionamento dall'interno che non sia solo mera critica e denuncia, ma concreta disponibilità di alternative e di dialogo con un settore, quello della tecnica, che fino a ieri sembrava irrimediabilmente separato dall'uomo" (Gatt 1964: 4). Gatt infine riteneva decisivo il fine sociale dell'artista che all'opposto

5 "Inoltre nel 1951 a Zagabria si è formato il gruppo artistico, chiamato, Studio Sperimentale 'Exat 51' [...] Tra i membri del gruppo, troviamo i nomi dell'architetto Vjenceslav Richter [...] e i pittori Ivan Picelj Aleksander Srnec e Vlado Kristl, di quest'ultimi nel 1953 venne ospitata una mostra di dipinti a [...] Belgrado. [...] Questa tradizione [...] ha creato una piattaforma adatta per la sua inclusione in un quadro internazionale più ampio [...]. Così oggetti d'arte scientifici perdono il loro carattere 'unico' e possono essere facilmente convertiti in prodotti meccanici, nella produzione in fabbrica, che influenza certamente la loro capacità di ampia diffusione con prezzi di vendita più bassi. [...] A condizione che si accettino queste nuove forme di espressione artistica, o chiamiamole come vogliamo, si deve cercare quindi il loro futuro nella sintesi con l'architettura". 
dell'artista borghese, la cui ambizione era il successo e il consenso del mercato, avrebbe dovuto essere più simile ad un ingegnere con il compito di edificare la società futura.

\section{LA STORICIZZAZIONE DELLA NUOVE TENDENZE COME CONTRO-RIVOLUZIONE}

Secondo il marxismo la produzione intellettuale nelle sue forme era determinata da quella materiale che a sua volta doveva essere considerata nella sua "forma specifica" (Marx \& Engels 1967: 214). Ne derivava che negli anni Sessanta la specifica forma del capitale era la tecnologia mentre l'arte poteva accettare o rifiutare tale presupposto. Tuttavia se l'arte diventava un fenomeno storico avrebbe subito un rapido processo di storicizzazione - o in negativo di obsolescenza - dovuto alle leggi del mercato. Il merito delle nuove tendenze fu di confrontarsi con il mercato e, purtroppo, di esserne fagocitate, ma in tal modo divennero chiari i limiti della collaborazione tra artista e industria.

Nel 1965 Nova tendencija 3 ospitò il convegno internazionale di Brezovica (Zagabria): un confronto sulle nuove modalità operative artistiche e sulle teorie estetiche derivate dalla tecnologia. Vennero presentati oggetti d'arte prodotti in serie come nel disegno industriale, opere-ambienti cinetici e optical e le prime elaborazioni grafiche realizzate con gli elaboratori elettronici. Secondo alcuni dei partecipanti, i risultati non furono del tutto soddisfacenti ma fu l'occasione per comprendere che le istituzioni culturali potevano interagire con la società in modo propositivo e non solo conservativo, e che gli artisti non volevano limitarsi a lavorare come ingegneri, pur impiegando i primi computer come un nuovo mezzo espressivo per la loro creatività.

\subsection{Il museo e la società tecnologia}

Dal convegno del 1965 emerse che il rapporto tra museo e industria, nella proposta di Palma Bucarelli, direttore della Galleria Nazionale d'Arte Moderna di Roma, si sarebbe attuato se gli artisti fossero stati impiegati nell'educazione alla visione del pubblico e avessero prodotto opere visuali divulgative come per esempio le stampe serigrafiche. Queste offrivano bassi costi di produzione, maggiore circolazione e stimolavano le capacità ottiche dell'osservatore. "Le nuove tendenze [...] si sottraggono [...] al mercato artistico [...]. In quanto il risultato di queste ricerche sono tracciati metodici e dimostrativi per l'educazione visiva, e in special modo per l'educazione visiva degli operatori industriali, il messo principale per il rapporto tra ricerca estetica e pubblico, [...], è il Museo d'Arte Moderna" (Bucarelli 1965: 21). 
La proposta incontrò il parere favorevole della rete museale jugoslava, considerando che Božo Bek, direttore della Galleria d'Arte Moderna di Zagabria, promosse la possibilità di organizzare una serie di conferenze sul tema espresso da Bucarelli, il cui intervento in traduzione venne allegato alla lettera che Miodrag Protić, direttore della Galleria d'arte moderna di Belgrado, ricevette da Zagabria ${ }^{6}$.

La questione della conservazione e divulgazione dell'arte era fortemente avvertito anche a Belgrado, considerando che nell'ottobre del $1965 \mathrm{fu}$ aperto al pubblico cittadino il Muzej Savremene Umetnosti (Museo d'arte contemporanea). Tra i collaboratori di Protić, direttore del museo, Đorđević si occupava di conservare l'arte jugoslava dopo il ' 45 e di collezionare le nuove tendenze per la prima volta a Belgrado: "Zasnivajući se na statičkim ili kinetičkim vizuelnim senzacijama, dekorativnom skladu bliskom industrijskom dizajnu i primenjenim rezultatima egzaktnih nauka [...] nove tendencije su se razvile u međunarodni pokret čiji je jedan od centara postao i Zagreb [...]" (Đorđević 1965: 44) ${ }^{7}$.

Musealizzare le nuove tendenze equivaleva a neutralizzare qualsiasi ambizione rivoluzionaria, pur lasciando ancora aperto il dibattito sul contrasto tra arte e tecnica nel tentativo di consolidare i risultati raggiunti.

\subsection{La non-ideologia e la fine dell'opposizione al sistema dell'arte}

Il problema che emerse riguardò la concreta ricaduta dell'ideologia della tecnica sul lavoro degli artisti. In Italia i critici d'arte avevano progressivamente constatato l'incoerenza di molte dichiarazioni nei manifesti delle nuove tendenze. Un diverso approccio verso la società cominciava ad essere richiesto all'artista e alla sua opera. Divenne evidente sia che molti artisti erano oramai entrati a far parte del sistema dell'arte capitalista, con mostre personali negli Stati Uniti (sotto l'etichetta Optical Art) e nei maggiori musei occidentali europei, sia che la stessa manifestazione era stata riassorbita dall'establishment della cultura ufficiale.

Già dal 1964 Argan aveva intuito le contraddizioni tra prassi e ideologia nelle nuove tendenze. Considerò l'arte ghestaltica come un 'terza via' - con evidente corrispondenza con il nuovo corso jugoslavo - mentre la Pop art fu biasimata come anarchia di destra, ma entrambe non erano effettive manifestazioni ideologiche:

${ }^{6}$ Archivio MSU, Zagreb. NT3, br. 89 od 251 do 699. Lettera di Božo Bek a Miodrag B. Protić, del 11/05/1965.

7 'Sulla base di sensazioni visive statiche o cinetiche, sull'armonia decorativa vicina al disegno industriale e sui risultati applicati delle scienze esatte [...] le nuove tendenze si sono evolute in un movimento internazionale di cui Zagabria è diventata uno dei centri [...]." 


\begin{abstract}
“Obbiettivamente i 'gruppi' ghestaltici oggi operanti non hanno risolto queste aporie; e corrono il rischio di ridurre la loro ricerca al progetto del progetto, della metodologia progettistica, della 'progettualità' [...]. La politica non è più attuale: l'operatore industriale non fa politica perché ha l'alibi della tecnica, l'opposto dell'ideologia [...]. La riduzione delle tecniche artistiche alla metodologia intenzionata del progettare non constituisce, in sé, la trasformazione radicale del processo artistico annunciata e promossa dalle avanguardie [...]. Ghestalt e Pop-Art sono due modi di arte non-ideologica, e non è la stessa questione; ma ci vuol poco a vedere che, se la Gestalt è tipicamente 'terza forza', la Pop-Art è anarchia di destra, qualunquismo reazionario" (Argan 1965: 47, 51).
\end{abstract}

Anche Apollonio non era in accordo con quanto stava accadendo tra le fila delle nuove tendenze e nello stesso periodo rilesse in modo strutturalista il rapporto tra uomo, natura e tecnica: "L'impresa artistica contemporanea [...] intende scoprire e, quindi, affermare l'intima struttura del reale. [...] la natura è in continuo divenire, è quella che meglio figura il progredire dallo stabile all'instabile senza smentire né l'uno né l'altro, perché è un complesso di elementi inseparabili [...]" (Apollonio 1966a: 20). La natura come struttura matematica poteva essere calcolata dai primi computer che sarebbero stati impiegati come mezzi di produzione artistica, non forzatamente esaltazione della tecnologia ma come estensione della creatività umana.

La capacità innovativa del rapporto artista-tecnologia fu sostenuta da Bihalji-Merin in una lettera del marzo 1966 dove, ringraziando Bek per il catalogo di Nova tendencija 3, ribadiva quanto fosse stato fondamentale per l'arte jugoslava avviare una ricerca che sarebbe stata feconda di sviluppi per le giovani generazioni di artisti: "Meni je jasno koliko je trebalo znanja, organizacione sposobnosti, upornosti i ličnog angažovanja da se, protiv svih otpora i teškoća ostvari ovo značajno delo, koje će se u našoj zemlji i u svetu proučiti u vezi sa svim traženjima kinetike, svetla i kibernetike u umetnosti". ${ }^{8}$

All'opposto un mutamento di prospettive stava avvenendo nella critica d'arte europea. Pochi mesi più tardi, nella Biennale di Venezia del 1966 Julio Le Parc vinse il premio per la pittura. L'opera di Le Parc rappresentava una via per fuggire l'impasse ideologica delle nuove tendenze. Se non erano più rivoluzionarie, potevano però sviluppare la creatività umana intesa come 'gioco'. Il critico d'arte Luigi Carluccio (1966) evidenziò un nuovo fattore: il passaggio ideologico dall'homo faber all'homo ludens. La tecnica quindi poteva essere umanizzata attraverso un rapporto, basato sul

${ }^{8}$ Archivio MSU, Zagreb. NT3, br. 89 od 251 do 699. Lettera di O. Bihalji-Merin a Božo Bek del 05/03/1966. "Capisco come sia necessario informare, organizzare le competenze, la perseveranza e l'impegno personale, contro ogni resistenza e difficoltà per realizzare questo lavoro in modo significativo, da cui verrà l'analisi delle relazioni tra tutti i conseguimenti dell'arte cinetica, ottica e cibernetica nel nostro paese e nel mondo.” 
processo ludico ed artistico e, come aveva spiegato Johan Huzinga (1949), ritrovando un'intenzionalità comune all'arte e alla scienza.

\section{LA NUOVA TENDENZA ITALIANA E IL SISTEMA DELL'ARTE A BELGRADO}

A Belgrado dal 1965, complice il nuovo museo d'arte contemporanea, si viveva il clima di una società proiettata nel futuro, in un momento di grande fiducia e apertura all'esterno. Protić nella monografia edita per promuovere il Muzej Savremene Umetnosti poneva l'accento sull'importanza che avrebbe avuto il museo nella diffusione e valorizzazione dell'arte sperimentale: "Ukratko, da omogući njeno dublje saznanje, njeno stalno prisustvo i zračenje, $[\ldots]$ njenog ontološkog i njenog teleološkog vida i uspostavi prekinut međuodnos i međuakciju: umetnik-delo-publika i uvede je u život društva i pojedinca, [..., ] saznanje i moral, podstakne stvaranje ličnih imaginarnih muzeja i umetnost vrati ljudima" (Protić 1965: 15)9.

Nel Muzej Savremene Umetnosti, la militanza di Jerko Denegri per la divulgazione delle nuove tendenze era stata decisiva e contribuì a trasformare la Galerija Doma Omladine in un centro artistico sperimentale di livello internazionale. Perché ciò avvenisse, nel novembre 1965, Denegri avvertiva, in modo simile a Bihalji-Merin e Protić, che l'arte dei giovani artisti belgradesi avrebbe dovuto aggiornarsi sui nuovi rapporti tra artista, società e tecnica:

"Stvarnost naše znanstvene i tehničke civilizacije u stanju je da pruži čovjekovom progresu ogromne i gotove beskrajne mogućnosti [...]. Potreba je i funkcija plastičkih umjetnosti u našem vremenu, ne da se tim tekovinama prkosno i bezrazložno opiru, ignorirajući time razvitak ljudske svijesti na ostalim područjima stvaralačke aktivnosti - znanosti, filozofiji i socijologiji suvremenog industrijskog društva - već da ih u granicama svojih spoznajnih i konstitutivnih mogućnosti prihvate, razviju i primjene u neposrednoj čovjekovoj životnoj sredini, [...], sredstva vizuelnih informacija i oblikovanja industrijskih predmeta široke potrošnje [...]" (Denegri 1965) ${ }^{10}$.

9 "Brevemente, per facilitare la sua conoscenza più profonda, la sua costante presenza e la irradiazione, [...] la sua visione ontologica e teleologica e stabilire una interrelazione e interazione interrotte: artista-opera-pubblico e portare nella vita della società e dell'individuo [...], la conoscenza e la morale, incoraggiare la creazione di un immaginario museo personale e aprire l'arte alla gente."

${ }^{10}$ La realtà della nostra civiltà scientifica e tecnica è in grado di fornire un vasto progresso umano e quasi infinite possibilità [...]. È necessaria anche una funzione delle arti plastiche nel nostro tempo, che non dipendono su questi risultati in modo provocatorio e irragionevole, ignorando in questi lo sviluppo della consapevolezza umana in altri settori di attività creative - scienza, filosofia e sociologia della moderna società industriale - ma 


\subsection{Luoghi: MSU e Galerija Doma Omladine}

Nell'aprile del 1966, grazie ad un accordo tra il governo italiano e quello jugoslavo, e alla collaborazione tra l'Ente Autonomo La Biennale di Venezia e il Muzej Savremene Umetnosti di Belgrado, venne allestita Artisti italiani d'oggi/Italijanska umetnost danas ${ }^{11}$, dove esposero le maggiori tendenze dell'arte italiana, dall'informale alla nuova figurazione, all'arte ghestaltica. Protić e Čelebonović presenziarono l'inaugurazione e le loro parole colpirono i rappresentanti dell'Ambasciata d'Italia ${ }^{12}$ :

"La mostra si è inaugurata a Belgrado il giorno 20 di aprile. Il direttore del Museo di Arte moderna, Miodrag Protić, ha salutato gli invitati [...] Il critico Aleksa Čelebonović ha espresso un giudizio altamente favorevole sulla mostra e, a suo avviso, gli stimoli che essa ha lasciato sugli artisti che l'hanno visitata non resteranno senza eco. E ciò pare ovvio, [...] perché gli artisti jugoslavi guardano e seguono oggi soprattutto i movimenti e le correnti artistiche italiane. [...] Non par escluso che la Mostra all'inizio abbia incontrato qualche difficoltà di interpretazione fra $\mathrm{i}$ critici, in parte sorpresi e in parte prudenti dinanzi a un tipo di arte che gli organi politici non hanno mai incoraggiato troppo."

Una mostra istituzionale che confermò, involontariamente, la fine dell'arte ghestaltica come arte rivoluzionaria. Parallelamente, con intenti più sperimentali, nella Galerija Doma Omladine esposero tra il 1966 e il 1967 alcuni artisti delle nuove tendenze, accompagnati dagli interventi critici di noti studiosi. I testi finalmente tradotti consentivano ad un pubblico più ampio di avvicinarsi alle nuove tematiche dell'arte contemporanea.

Nel dicembre 1966, per la mostra Galleria del Deposito, Germano Celant introduceva le opere di Jesus Rafael Soto, riprendendo alcune idee di Apollonio e sostenendo l'origine storica delle nuove tendenze: "Ovaj zahtev odnosi se i na 'ne-predmetne' slikare [...] u čijim su se slikama sadržavale relacije određenih odnosa prirodnog ili metafizičkog reda. Sa istorijskog aspekta prihvatili smo gledište da je pozivanje na procese ili spoljašnje

che accettino entro i limiti della loro capacità cognitiva e costitutiva, lo sviluppo e l'applicazione nell'ambiente umano [...] dei mezzi di informazioni visive e della progettazione dei beni di consumo industriali."

${ }^{11}$ Archivio Biblioteca MSU, Beograd. È conservata copia del catalogo stampato per l'occasione in lingua serba.

${ }^{12}$ Archivio ASAC, Venezia, Fondo storico. Serie arti visive, Unità 198. Mostre d'arte italiana all'estero, Artisti italiani d'oggi, Belgrado 20 aprile - 10 maggio 1966. Telespresso n. 2800/1080 inviato dall'Ambasciata d'Italia a Belgrado al Ministero degli Esteri e all'Ente Autonomo La Biennale di Venezia. 
vrednosti u prirodi bilo samo "popularisanje sadržajnih i senzibilnih pojava"" (Celant 1966) $)^{13}$.

Argan nel testo per la mostra Drei, Glattfelder, Guerrieri del marzo 1967 riprendeva il discorso fatto per il convegno di Rimini del 1964, ribadendo il suo interesse per l'impegno jugoslavo verso un'arte visuale capace di accogliere le istanze della tecnica allo stesso modo di quelle sociali: "Siguran sam da će njihova istraživanja biti lako prihavaćena u Jugoslaviji, gde su analogna ispitivanja bila vođena u različitim pravcima pokrećući izložbe i rasprave od najveće važnosti, i pretpostavljam da će ova izložba moći doprineti produbljivanju one internacionalne saradnje na planu projektovanja vizuelnih struktura za kojima se sve više ukazuje neophodna potreba" (Argan 1967) ${ }^{14}$.

Infine, nell'aprile 1967, la mostra Getulio Alviani chiuse la serie dedicata alle ricerche sperimentali italiane, introducendo allo stesso tempo le più recenti analisi critiche di Carla Lonzi che ribaltavano il senso di un'arte tecnolgica in chiave non esistenziale e non ideologica. "Esencijalno da bi se živelo u skladu sa činjenicama - bez rizika da se pogreši - i ne predlagati nijednu ideologiju; [...] on je savršeni antipod u odnosu na ovog koji želi da vidi potvrđenu u umetnosti nužnost svojih drama [...]" (Lonzi 1967)15.

\subsection{Parole: Umetnost dal 1965}

Accanto alle attività di Dom Omladine sempre nel 1965 la nuova rivista Umetnost - nella cui redazione figuravano Čelebonović, Trifunović, Protić e Denegri - permise l'incontro della critica serba con quella italiana e croata (Putar 1965; Meštrović 1965) sui temi dell'arte e della tecnologia.

Il primo numero della rivista, nel gennaio 1965, riproduceva in copertina un'opera astratto geometrica di Radomir Damnjanović Damnjan e nel suo intervento Protić rivendicava la spinta ideologica di umanesimo e socialismo nel decifrare la relazione arte-società: "Zato nova umetnost ne

13 “Questo requisito si applica ai pittori 'non oggettivi' [...] in cui le immagini contengono le relazioni di alcuni rapporti di ordine naturale o metafisico. Storicamente abbiamo accettato che il riferimento ai processi o ai valori esterni alla natura fosse 'una divulgazione dei contenuti e dei fenomeni sensibili'."

14 "Sono sicuro che sarà facile per la loro ricerca essere accettata in Jugoslavia, dove un analogo studio è stato condotto in diverse direzioni allestendo mostre e discussioni della massima importanza, e credo che questa mostra sarà in grado di contribuire all'approfondimento della cooperazione internazionale nel campo delle strutture di visual design che sta diventando necessaria."

15 "Essenzialmente si dovrà vivere conformemente ai fatti - senza il rischio di un errore - e non proporre alcuna ideologia; [...]. Egli è l'antipodo perfetto in rapporto a chi vuole vedere confermata nell'arte la necessità del proprio dramma [...]." 
može da zaobiđe industriju niti moderno društvo to zaobilaženje može da dopusti. [...] Putevi od umetnosti do života su dakle složeni, ali nezaobilazni ukoliko želimo da socijalizam ne bude samo ekonomska i politička kategorija, već i kategorija humanizma i kulture - pravi socijalizam" (Protić 1965b: 9, 10) ${ }^{16}$.

A livello ideologico si tentava di decifrare un nuovo umanesimo, in una visione non così lontana da quella sostenuta da Argan. Inoltre, a livello terminologico cresceva la fortuna del termine "ghestaltico", come nel 1965 ebbe a scrivere Čelebonović, su Umetnost: "Kada su u pitanju geometrizirane konstrukcije, savršeni tehnički sastavi i kontrolisana mehanika kretanja u delima istraživača 'gestaltične umetnosti' očigledno se više ne može govoriti o netaknutoj prirodi stvari, jer su ta dela autori već po metodologiji svog rada smislili, u celosti predvideli i izveli. [...] Gestaltična dela su katkada veoma bliska industrijskom 'dizajnu' i nesumnjivo s ovim imaju interferencija" (Čelebonović 1965: 17) ) $^{17}$.

Tra storia e attualità, nel gennaio del 1966 su Umetnost Denegri (1966) presentò il gruppo Exat51 e vennero tradotte le trascrizioni del convegno di Brezovica, in cui Apollonio aveva esposto le proprie perplessità sugli effettivi risultati raggiunti dalle nuove tendenze. Era posta in discussione la loro assoluta novità e mostrato che le opere esposte avevano perso il reale rapporto con le dinamiche della società contemporanea (Nova tendencija 3 1965).

L'anno successivo si continuò a perseverare sull'argomento pubblicando l'articolo di Apollonio (1966b, 1967), traduzione dell'originale Arte programmata, e intitolato Nova tendencija u Italiji: "L'esperienza in atto con le prove della così detta arte programmata o cinetica o ghestaltica o di 'nuova tendenza' [...] è la logica conseguenza di un filone di ricerca [...] che soltanto gli ostacoli di un dibattito fra differenti posizioni ideologiche e di linguaggio hanno potuto deviare o arrestare [...]" (Apollonio 1966b: 54).

Eppure la nuova tendenza poteva avere ancora credito in Jugoslavia a differenza dell'Italia. La diversa traduzione del titolo, in modo più accattivante per il pubblico belgradese, evidenziava infatti una liaison ideale

16 "Perché la nuova arte non può evitare l'industria né si può permettere di aggirare la società moderna. [...] I passaggi dall'arte alla vita sono quindi complessi, ma inevitabili se vogliamo che il socialismo non sia solo una categoria economica e politica, ma anche una categoria umanistica e culturale - il socialismo reale."

17 "Quando si tratta di costruzioni geometriche, perfetto disegno tecnico e controllato movimento meccanico nelle opere dei ricercatori d'arte 'ghestaltica', ovviamente non si può più parlare di una natura incontaminata, perché gli autori hanno già concepito queste opere nella metodologia del loro lavoro, già previsto nell'insieme e poi eseguito. [...] Le opere ghestaltiche sono a volte molto vicine al 'design' industriale e senza dubbio tra di loro hanno interferenze." 
tra Italia e Jugoslavia. Inoltre l'articolo cadendo nel periodo della Treći Trijenale Likovnih Umetnosti, da luglio a settembre 1967, poteva leggersi come propedeutico a comprendere la linea della nuova tendenza.

Nella rassegna erano state esposte le più attuali espressioni dell'arte jugoslava, in modo che anche le nuove tendenze figurassero nel panorama artistico istituzionale. Il premio per la scultura fu assegnato a Vojin Bakić, che era il rappresentate della nuova tendenza croata nella sezione curata da Boris Kelemen (1967).

$\mathrm{Su}$ Umetnost tutto ciò ebbe ampio spazio. Le ragioni storiche e di confronto con l'attualità europea furono indagate da Bihalji-Merin, mentre Denegri si occupò della specifica situazione jugoslava.

Bihalji-Merin nel suo articolo, oltre ad impiegare l'intero lessico riferibile all'arte cinetica e ghestaltica, dimostrava di essere in sincronia con i nuovi orientamenti interpretativi che la critica italiana andava applicando alla lettura delle opere delle nuove tendenze: "Lumino-dinamična umetnost može kao kibernetski manirizam da posluži potrebi modernog homo ludens-a za zabavom, pomoću usmerenih efekata proizvedenih čudnovatim automatima da pruži monumentalnu predstavu mašinskog folklora dvadesetog veka" (Bihalji-Merin 1967: 12) ${ }^{18}$.

Denegri, da parte sua, entrava nel dettaglio delle opere presentate sotto l'etichetta di "nova tendencija" e le introduceva riportando le parole di Argan, riguardanti la metodologia della ricerca di nuova tendenza: "Ovi objekti, [...], mogu zadovoljiti onu strogu metodsku oznaku 'istraživanja kao načina mišljenja' (Argan), zato što polaze od jedne egzaktne polazne pretpostavke koja treba da kroz sâm proces rada na djelu bude verificirana kao moguće i realno potvrđenje problema podvrgnutog određenoj eksperimentalnoj temi" (Denegri 1967: 33) ${ }^{19}$.

I succitati brani indicavano quanto l'ambiente culturale italiano e serbo fossero in reciproco dialogo, in modo nuovo per l'epoca e foriero di interessanti sviluppi nel riscrivere il rapporto tra arte e tecnologia nell'arte contemporanea.

18 'L'arte ottico-cinetica può essere un manierismo cibernetico per soddisfare i bisogni di divertimento dei moderni homo ludens, con l'aiuto dell'effetto di creazioni realizzate con sbalorditivi meccanismi per offrire una monumentale idea del folklore della macchina del XX secolo."

19 “Questi oggetti, [...], sono in grado di soddisfare una severa indicazione di metodo che è 'la ricerca come indagine' (Argan), perché presumono un'esatta ipotesi di partenza che dovrebbe, attraverso il solo processo che agisce nell'opera, essere la verifica e la conferma possibile e reale del problema esposto in un determinato soggetto dell'esperimento." 


\section{VERSO UNA STORIA CONDIVISA DELLA CRITICA D'ARTE}

Dopo il 1967 artisti e studiosi d'arte italiani, avvicinandosi alle teorie di Herbert Marcuse (1967/1968) e Jurgen Habermas (1968/1969), esercitarono una contro-azione critica sul pensiero scientifico e tecnologico. Come nel caso di Germano Celant e dell'Arte povera, la critica militante della nuova sinistra considerò le nuove tendenze complici di esposizioni "borghesi" come Nuove tecniche d'immagine (Celant 1967) e succubi dell'industria e del capitale.

In Italia, quindi, sarebbe tramontata per sempre l'idea di un progresso tecnologico che avvicinava le nuove tendenze al radicalismo marxista. E una volta che le nuove tendenze furono ufficializzate, il futuro era altrove. A Zagabria si sarebbe approfondito il rapporto tra nuove tendenze e i computer fino al 1973. Mentre a Belgrado alla soglia del 1970, sarebbero esplosi i nuovi fermenti artistici, rivelando le idiosincrasie espresse dal rapporto tra cultura, economia e politica, e maturate dopo l'autunno del '68 (Protić 1969).

Tracciare un bilancio ed una storia delle relazioni tra critica d'arte italiana e serba dal 1957 al 1967 richiede ancora lunghe analisi e studi. Dagli esempi riportati, tuttavia, si possono trarre alcune considerazioni di carattere generale.

In primo luogo, gli scambi culturali tra Italia e Serbia, grazie anche agli studiosi croati, furono favoriti dai cambiamenti sociali e, nei limiti delle diverse economie, dalla tecnologia che stava migliorando il tenore di vita quotidiano. In secondo luogo, i primi anni Sessanta segnarono un progressivo interessamento, in entrambi i paesi, alle ricerche artistiche legate all'informale e all'arte ghestaltica. L'arte ghestaltica permise una riflessione di natura ideologica sul modo in cui il ruolo dell'arte nell'industria e nel socialismo si fosse oggettivato nelle opere degli artisti italiani e jugoslavi.

In modo particolare gli scritti di Protić, Čelebonović, Denegri e Bihalji-Merin autonomamente e in relazione con quelli di Argan, Apollonio, Bucarelli e Gatt, agitarono e condivisero il comune ambiente della critica d'arte croata, che permise all'arte di entrambe le nazioni di ridurre, e forse di superare, l'apparente divario culturale che all'epoca della Guerra Fredda aveva contrapposto il mondo occidentale a quello orientale. 


\section{BIBLIOGRAFIA}

AA. VV. (1965). Nova tendencija 3. Umetnost, 5, 69-75.

Apollonio, U. (1963a). Ipotesi intorno a una nuova linguistica. In Nuova Tendenza 2. Catalogo. (pp. non numerate). Fondazione Querini Stampalia. Venezia: Lomborso editore.

Apollonio, U. (1963b). Ipotesi su nuove modalità creative. Quadrum, 14, 5-34.

Apollonio U. (1966a). Sistema matematico e ordine naturale. Lineastruttura, $1,18-23$.

Apollonio, U. (1966b). L'arte programmata. Siprauno, 3, 54-67.

Apollonio, U. (1967). Nova tendencija u Italiji, Umetnost, 11, 6-3.

Argan, G. C. (1953, gennaio). A chi attende il comando?. Civiltà delle macchine, 1, 31-32.

Argan, G. C. (1959). Materia, tecnica e storia dell'informale. La Biennale di Venezia, 35, 2-7.

Argan, G. C. (1961). Salvezza e caduta dell'arte moderna. Il Verri, 3, 4-30.

Argan, G. C. (1963a, 24 agosto). La ricerca gestaltica. Il Messaggero, p. 3.

Argan, G. C. (1963b). Il rappporto arte-società nella condizione storica attuale. De Homine, 5-6, 104-109.

Argan, G. C. (1964, ottobre). Tecnica e ideologia in un Convegno a Rimini. Le Arti, 10, 32-33.

Argan, G. C. (1965). Progetto e destino. Milano: Il Saggiatore.

Argan, G. C. (1967). [senza titolo]. In Drei, Glattfelder, Guerrieri (1967).

Catalogo. (pp. non numerate). Beograd: Galerija Doma omladine.

Argan, G. C. \& Ponente, N. (1958/1959). Italia. In W. Grohmann (a cura di), L'arte dopo il 1945. La pittura (pp. 87-92). Milano: Il Saggiatore.

Bense, M. (1960). Aesthetica IV: Programmierung des Schönen. Krefeld/ Baden-Baden: Agis.

Bihalji-Merin, O. (1962). Prodori moderne umetnosti. Beograd: Nolit.

Bihalji-Merin, O. (1958/1959). Jugoslavia, Polonia. In W. Grohmann (a cura di), L'arte dopo il 1945. La pittura (pp. 132-138). Milano: Il Saggiatore.

Bihalji-Merin, O. (1967). Vreme, svetlost, pokret. Umetnost, 12, 5-14.

Bihalji-Merin, O. (1970). La fin del'Art à l'ère de la Science?. Bruxelles: La Connaissance S.A.

Bricco, P. (2005). Olivetti prima e dopo Adriano. Industrie cultura estetica. Napoli: L'ancora del Mediterraneo.

Bucarelli, P. (1965). In Nova tendencija 3. Catalogo. Galerija suvremene umjetnosti, Muzej za umjetnost i obrt, Centar za industrijsko oblikovanje (edizione internazionale, p. 21). Zagreb: MSU. 
Carluccio, L. (1966, 8 giugno), Gioco, passatempo e varietà sono protagonisti nel grande spettacolo della Biennale di Venezia. Gazzetta del Popolo, p. 6.

Celant, G. (1966). Jesus Rafael Soto. In Galleria del Deposito. Catalogo (pp. non numerate). Beograd: Galerija Doma omladine.

Celant, G. (1967). Nuove tecniche dell'immagine. Casabella-Continuità, 319, 59-62.

Čelebonović, A. (1965). Novi oblici u posleratnom slikarstvu. Umetnost, 2, $5-17$.

Denegri, J. (1965). Za jednu umjetnost gradjenja. In Anale Mladih, 1. Catalogo. (pp. non numerate). Beograd: Galerija Doma omladine.

Denegri, J. (1966). Ivan Picej. Jedan od inicijatora grupe EXAT 51. Umetnost, 5, 21-32.

Denegri, J. (1967). „Nove tendencije” na III trijenalu. Umetnost, 12, 33-34.

Denegri, J. (2001). EXAT 51 - Nove tendencije-umjetnost konstruktivnog pristupa. Zagreb: Horetzky.

Denegri, J. (2003). Inside or Outside "Socialist Modernist"? Radical Views on the Yugoslav Art Scene, 1950-1970. In D. Djurić \& M. Šuvaković (a cura di), Impossible Histories. Historical Avant-Gards, Neo-avant-gardes, and Post-avant-gardes in Yugoslavia, 1918-1991 (pp. 170-209). London: MIT Press.

Denegri, J. (2009). Jedna moguća istorija moderne umetnosti. Beograd kao internacionalna umetnička scena 1965-2006. Beograd: Signature, BIGZ, Muzej savremene umetnosti.

Denegri, J. \& Koščević, Ž. (1979). EXAT 51, 1951-1956. Zagreb: Galerija Nova. Devos, R. \& De Kooning, M. (2006). L'architecture moderne à l'Expo 58. Bruxelles: Fonds Mercator et Dexia Banque.

Dorfles, G. (1961). Ultime tendenze dell'arte oggi. Milano: Feltrinelli.

Đorđević D. (1963, 21 settembre). Nove tendencije 2. Borba, p. 6.

Đorđević, D. (1965). Slikarstvo posle 1945. In M. Protić (a cura di). Muzej savremene umetnosti (pp. 38-48). Beograd: MSU.

Egbert, D. D. (1970). Social Radicalism and the Arts - Western Europe A cultural History from the French Revolution to 1968. New York: Alfred Knopf.

Feierabend, V. \& Meloni, L. (2009). Gruppo N. Oltre la pittura, oltre la scultura, l'arte programmata. Milano: VAF - Foundation, Silvana Editoriale.

Gatt, G. (1964, settebre-ottobre). Arte, tecnica e ideologia. Il Sestante letterario, 5, 3-6.

Giedion, S. (1948). Mechanization takes Command. A Contribution to Anonymous History. Oxford: Oxford University Press. 
Habermas, J. (1968/1969). Teoria e prassi nella società tecnologica. Bari: Laterza.

Huzinga, J. (1949). Homo ludens. London: Routledge and Kegan Paul.

Jeannesson, S. (2002/2003). La guerra fredda. Una breve storia. Roma: Donzelli.

Kelemen, B. (1967). Nova tendencija. In Treći Trijenale Likovnih Umetnosti. Catalogo (pp. 107-114). Beogradski sajam. Beograd: Kultura.

Lonzi, C. (1967). [senza titolo]. In Getulio Alviani. Catalogo (pp. non numerate). Beograd: Galerija Doma omladine.

Marcuse, H. (1967/1968). La fine dell'utopia. Bari: Laterza.

Marx, K. \& Engels, F. (1967). Scritti sull'arte. Bari: Editori Laterza.

Medosh, A. (2010). Overcoming alienation/new tendencies 1961-1973. In C. Höller (a cura di), L'Internazionale - Post-War Avant-Gardes Between 1957 and 1986 (pp. 251-260). Zurich: JRP/Ringier.

Meneguzzo, M. (2012). Arte programmata cinquant'anni dopo. Milano: Johan\&Levi.

Merenik, L. (2010). Umetnost i vlast. Srpsko slikarstvo 1945-1968. Beograd: Vujačić kolekcija - Univerzitet u Beogradu, Filozofski fakultet.

Meštrović, M. (1965, aprile-giugno). Polifikacija vizuelnog - fenomen industrijske civilizacije. Umetnost, 2, 132-138.

Mumford, L. (1952). Art and Technics. New York: Columbia University Press.

Neumeyer, A. (1964). The research of meaning in modern art. New York: Prentice Hall.

Olivetti, A. (1959). La città dell'uomo. Milano: Comunità.

Piotrowski, P. (2009), In the shadow of Yalta. Art and the Avant-garde in Eastern Europe, 1945-1989. London: Reaktion Books.

Protić, M. (1965a). Upravnik Muzeja savremene umetnosti. In M. Protić (a cura di) Muzej savremene umetnosti (pp. 4-15). Beograd: MSU.

Protić, M. (1965b, gennaio-marzo). Od umetnosti do života. Umetnost, 1, $5-10$.

Protić, M. (1969, aprile-settembre). Sedma decenja kulturno-političke marginalije. Umetnost, 18-19, 5-10.

Putar, R. (1965, aprile-giugno). Nova tendencija 3. Umetnost, 2, 131.

Read, H. (1961). Art and Industry. New York: Horizon Press.

Rosen, M. (a cura di). (2010). A Little-Known Story about a Movement, a Magazine, and the Computer's Arrival in Art: New Tendencies and Bit International 1961-1973. Karlsruhe: ZKM.

Sauvage, T. (1957). Pittura italiana del dopoguerra. Milano: Schwarz editore.

Trifunović, L. (1990). Studije, ogledi, kritike. Vol. 4. Beograd: Muzej savremene umetnosti. 
Vrsaj, E. (1970). La cooperazione economica Italia-Jugoslavia. Trieste: Edizioni Rivista "Mladika".

Wachtell, A. B. (1998). Making a Nation, Breaking a Nation. Literature and culturale politics in Yugoslavia. California: Stanford University Press.

\author{
WRITING THE FUTURE: 1957-1967. TECHNICS \\ AND IDEOLOGY THROUGH THE CRITIC OF ART BETWEEN \\ ROME AND BELGRADE
}

\title{
Summary
}

From 1957 to 1967 several circumstances both in the Italian and the former Yugoslav Art World were related to the industrial system and to the so-called "ideology of technics". Marxist theories of social development met the reletionship between the humans and the machine. A common thinking on the technology was shared between artists and scholars who attempted to explain the meaning of Optical art. Particularly, the Italian critics of art, e.g. Giulio Carlo Argan and Umbro Apollonio, and the Serbian ones, e.g. Oto Bihalji-Merin and Jerko Denegri, took an interest in the exchanges between kinetic art and technics. Thanks to their Croatian colleagues, this situation was showed during the Zagreb international exhibitions called Nove tendencije and also it became evident by the new objects of the industrial design. The Italian and Serbian critics ambitously tried to picture as revolutionaries the contemporaneous new tendencies, whereas the latter was failing to face the art market's influences. Concerning the words and the language that have been expanded on catalogues and magazines, we could assume that there have been similarities between the two groups of critics, but actually today there is a lack of studies. This paper does not aim to complete the matter but to spark the historical and artistic debate.

Keywords: Technics, Ideology, Optical Art, Industrial Design, Nove tendencije, Critic of Art 